\title{
Una riflessione sull'efficacia delle politiche preventive per il controllo dei consumi di bevande alcoliche
}

\author{
Allaman Allamani*, Franca Beccaria**, Corrado Celata ${ }^{\circ}$, Francesco Cipriani $^{\circ \circ}$, \\ Shlomo Stan Einstein $\$$, Pasquale Pepe*, Franco Prina ${ }^{\wedge}$, Sara Rolando**, Fabio Voller*
}

\section{Diversità e aspetti comuni dei modi di bere e dei danni alcol-correlati}

Gli studi che qui passiamo in rassegna si riferiscono soprattutto, anche se non solo, ai paesi europei. Infatti l'Europa è un buon terreno di indagine in quanto caratterizzata storicamente da una elevata produzione e commercializzazione di bevande alcoliche (un quarto del contenuto alcolico nel mercato globale delle bevande alcoliche è di provenienza europea) e di consumo delle medesime (il quale in Europa è più del doppio della media mondiale).

Oltre a ciò, i popoli dei vari paesi europei attribuiscono diversi valori, funzioni e significati al bere, il che differenzia le loro attenzioni e preoccupazioni circa le bevande alcoliche, nonché le loro politiche alcologiche (Lolli, 1959; Sulkunen, 1989; Heath, 1995; Beccaria et al., 2012; Anderson e Baumberg, 2006; Møller e Anderson, 2012).

Infatti, mentre nelle culture del bere delle regioni settentrionali - paesi scandinavi e Regno Unito - vi sono aspettative positive sulla perdita del controllo e sull'ubriachezza, all'opposto nei paesi delle regioni meridionali e centrali - quali Spagna, Francia, Italia e Portogallo, e Svizzera, Austria e Germania meridionale - bere si combina col socializzare avviene in occasioni conviviali e sociali, usualmente ai pasti, e l'ubriachezza è vista in modo negativo (Järvinen e Room, 2007). Inoltre il consumo pro capite ha presentato trend specifici per ciascun paese.

Per esempio, per diversi anni dopo la fine della seconda Guerra Mondiale, Francia e Italia sono stati i maggiori consumatori, specie di vino: nel periodo dal 1961 al 1980 il consumo francese pro capite oscillava tra i 19 e i 26 litri di alcol puro per abitante dai 15 anni in su maggiore di 14 anni, quello italiano tra i 16 e i 19 litri.

Se prendiamo invece come punto di osservazione il 2008 , i più grandi consumatori erano gli austriaci $(12,4$ litri) e gli ungheresi (10,7 litri) (World Health Organization, 2011a).

Secondo lo studio AMPHORA, tra gli anni '60 e il 2000 il consumo alcolico nelle regioni europee meridionali è sceso da 19,39 a 10,39 litri di alcol puro per abitante maggiore di 14 anni, mentre nelle regioni dell'Europa centrale è aumentato

\footnotetext{
* Agenzia Regionale di Sanità, Firenze.

** Eclectica, Torino.

- ATS - Agenzia Tutela Salute della Città Metropolitana di Milano.

${ }^{\circ}$ Epidemiologia, Azienda USL Toscana Centro.

$\S$ New York University.

${ }^{\wedge}$ Università di Torino.
}

da 10,27 a 11,54 litri e nelle regioni dell'Europa settentrionale da 5,32 a 8,63 litri (Allamani, Pepe e Voller, 2015).

Inoltre, ad esempio, nel 1990 nella Federazione Russa si è registrato un consumo di 7,73 litri di alcol puro pro capite per abitante maggiore di 14 anni, che è salito a 10,12 litri nel 2014, mentre in Italia è rispettivamente disceso da 10,95 a 7,65 litri (European Health For All Database, 2016).

Dal punto di vista dei danni alcol-correlati si sono potuti osservare notevoli cambiamenti temporali (World Health Organization, 2015).

In Europa, la mortalità per patologie epatiche e quella per incidenti stradali - indicatori del danno alcol-correlato rispettivamente acuto e cronico - sono diminuiti tra gli anni '70 e gli anni 2000.

In 12 Paesi europei i valori della mortalità epatica sono diminuiti da 16,2 (negli anni 1970-1979) a 13,4 (negli anni 20002009) su 100.000 abitanti (Allamani, Pepe e Voller, 2015).

Ma questa diminuzione risultava dalla combinazione tra un ampio calo della mortalità $(65 \%)$ nelle regioni meridionali e un suo importante aumento nelle regioni settentrionali (39\%) e centrali (19\%) d'Europa.

D'altro lato, gli incidenti mortali sono scesi da 16,32 (19801989) a $8,7(2000-2009)$ su 100.000 abitanti (Allamani, Pepe e Voller, 2015).

Similmente, il tasso di decessi alcol-attribuibili (che include anche cancro e patologia cardiovascolare) sul totale dei decessi per il periodo 1990-2014 in tutti i Paesi europei, è globalmente aumentato del $4 \%$ e risulta soprattutto dalla combinazione delle riduzioni dei decessi nelle regioni mediterranea $(-27 \%)$, centroccidentale $(-25)$ e centrorientale $(-15 \%)$ e dei loro aumenti nell'area orientale $(+22 \%)$ e sudorientale (+65\%) della Regione Europea dell'OMS (Schütze et al., 2011; Shield, Parkin et al., 2016; Shield, Rylett e Rehm, 2016).

2. Le misure di politica alcologica e il loro impatto sul consumo alcolico e sui danni alcol-correlati

Nei paesi del nord Europa, così come in nord America, esistono già da molti decenni preoccupazioni per i danni alcolcorrelati e, ai fini di ridurli, si sono introdotte politiche preventive per il controllo del consumo alcolico.

Sull'efficacia di tali politiche il dibattito è ora aperto.

Senza dubbio molti studi degli ultimi 30 anni ne hanno dimostrato l'efficacia. 
Sono state oggetto di studio le normative su limitazioni al bere di chi guida, tasse (accise e IVA), determinazione dei prezzi e del prezzo minimo per unità di bevanda alcolica, età minima per l'acquisto, limiti alla disponibilità-accessibilità dei luoghi di vendita, controllo della pubblicità e del marketing, nonché programmi di informazione ed educazione sanitaria, azioni di comunità, interventi brevi su bevitori a rischio che non hanno avuto trattamento specialistico (Anderson et al., 2009; Babor et al., 2010; Anderson et al., 2012).

Sulla base di queste evidenze, organismi internazionali ed esperti hanno promosso linee guida specifiche.

In particolare sono stati definiti come "best buys", cioè "affari migliori" per le nazioni che dovrebbero dotarsi di politiche alcologiche, l'aumento delle tasse, restrizioni alla vendita al dettaglio e bandi alla pubblicità delle bevande alcoliche (World Health Organization, 1993, 2011 b, 2014 a; Commissione Europea, 2010; World Health Organization \& World Economic Forum, 2011; Rehm \& Scafato, 2011; Anderson et al., 2012, Rehm et al., 2015).

A sostegno dell'efficacia delle norme contro la guida in stato di ebbrezza, una revisione sistematica di 76 studi internazionali pubblicati tra gli anni '70 e '90 dimostra la riduzione degli incidenti stradali e dei decessi che coinvolgono i veicoli a motore in associazione con norme limitanti il tasso alcolemico alla guida e l'introduzione di punti di controllo della sobrietà e di alcol-test a campione (Shults et al., 2001).

Utilizzando dati europei sugli incidenti stradali del periodo 1991-2003, un altro studio ha dimostrato che la riduzione dei limiti di tasso alcolemico si è rivelata efficace nella diminuzione dei decessi quando accompagnata da controlli a campione su strada (Albalate, 2006).

In una ricerca che ha analizzato una serie di dati australiani fino al 2006, l'introduzione degli alcol-test a campione (unitamente alla legislazione sull'uso delle cinture di sicurezza) è risultata associarsi a diminuzioni significative nella mortalità da incidenti stradali, specie per i giovani maschi (Jiang et al. 2015).

Inoltre, uno studio condotto sui paesi dell'Europa meridionale ha mostrato che le restrizioni alla guida in stato di ebbrezza si sono rivelate uno dei fattori correlati alla riduzione degli incidenti mortali (Allamani, Pepe et al., 2014).

Vi sono solide evidenze dell'efficacia delle misure che, tramite tasse e controllo dei prezzi, regolano il mercato delle bevande alcoliche.

Numerose ricerche documentano che quando tasse e prezzi delle bevande crescono o diminuiscono, il bere ad alto rischio e i danni alcol-correlati mostrano un andamento opposto (Chaloupka et al., 2002; Waagenar et al., 2009; Elder et al., 2010; Patra et al., 2012; Zatonski et al., 2015).

Inoltre si è recentemente stimato che ove si applicasse, anziché la tassazione attuale basata sul valore del prodotto, una tassazione basata sul contenuto di alcol, cioè un prezzo unitario minimo, ci sarebbe un maggior impatto sul consumo dannoso, con effetti minimi sui consumatori moderati (Meier et al., 2016).

L'introduzione di un'età minima di legge per l'assunzione di alcol si è dimostrata efficace, con una relazione inversa tra consumi alcolici e incidenti stradali (Shults et al., 2001; Wagenaar e Toomey, 2002; McCartt et al., 2010).
In letteratura alcune fonti riportano l'efficacia protettiva delle politiche restrittive sul consumo di bevande alcoliche (aumento di tasse e prezzi e abbassamento dell'età minima per l'acquisto di bevande alcoliche) nei confronti dei tassi di suicidio (Pridemore et al., 2009, 2013; Xuan et al., 2016).

Limitazioni alla disponibilità di, e accessibilità a, luoghi e orari di vendita sono state dimostrate associarsi a minori consumi alcolici e a minori danni alcol-correlati (Gruenewald, 2011; Wicki e Gmel, 2011; Kypri et al., 2011; Cook et al., 2014; Kolosnitsyna et al., 2014), mentre l'estensione degli orari e l'aumento di densità dei punti vendita aumentano $\mathrm{i}$ danni associati al bere (Stockwell \& Chikritzhs 2009; Hahn et al., 2010; Lippy et al., 2014; Xuan et al., 2015).

In modo opposto, tuttavia, Green, Heywood e Navarro (2014) hanno rilevato un'associazione tra l'estensione degli orari di chiusura dei bar in Inghilterra e in Galles e una diminuzione degli incidenti stradali, specie tra gli automobilisti giovani.

Esistono alcune ricerche sulle conseguenze che la pubblicità ha sui consumatori, specie giovani (de Bruijn et al., 2016).

Ma non vi sono ancora evidenze circa l'efficacia delle misure che la limitano, anche se vi è un ampio consenso sull'effetto positivo che esse possono aver avuto con la loro introduzione in Norvegia nel 1975-77 e in Francia nel 1991 (legge Evin) (Wilkinson e Room, 2009; De Bruijn et al., 2010; Anderson et al., 2012; Monteiro et al., 2017).

Parecchi studi sono stati compiuti per documentare l'efficacia e la relazione costo/efficacia degli interventi brevi sui bevitori ad alto rischio (Anderson et al., 2012; Angus et al., 2014; Babor et al., 2017) che tuttavia, altre ricerche hanno negato, stabilendo la non efficacia degli stessi (Foxcroft et al., 2014). Una posizione intermedia è rappresentata dall'analisi di Grant e collaboratori (2016) che conclude per effetti modesti, ma comunque positivi e potenzialmente significativi, degli interventi in questione.

Infine, i programmi educativi nelle scuole e a livello di comunità, per quanto difficili da valutare dal punto di vista dell'efficacia immediata, appaiono però necessari almeno per sostenere e attuare localmente una politica di prevenzione basata su più componenti (Treno et al., 2007; Foxcroft e Tsertsvadze, 2011; Anderson et al., 2012).

\section{Discordanze tra gli effetti attesi dalle politi- che alcologiche introdotte e i cambiamenti effettivi nei consumi e nei danni}

Nonostante le numerose evidenze scientifiche, parte della recente letteratura sta segnalando una sostanziale variabilità nell'efficacia delle misure di politica alcologica e, in certi casi, addirittura una loro inefficacia o efficacia negativa, con effetti opposti riguardo le aspettative.

Anzitutto lo studio AAA-Prevent finanziato dall'UE, con 34 partecipanti rappresentanti 17 Paesi europei, è giunto alla conclusione che, poiché i modi di consumo di bevande alcoliche variano secondo i paesi, mentre non cambiano significativamente da un paese all'altro le politiche alcologiche di controllo, queste ultime non sono in grado di spiegare le diversità nel consumo alcolico che si riscontrano all'interno di ciascun paese (Berten et al., 2013). 
Uno studio che ha utilizzato dati dell'OCSE, provenienti in prevalenza da paesi europei, durante il periodo dal 1975 al 2000, ha dimostrato:

(a) la non efficacia della proibizione della pubblicità sulla riduzione dei consumi e

(b) l'assenza di una significativa risposta di mercato alle vendite promosse dalla pubblicità (Nelson, 2010).

Un altro studio ha dimostrato che con la diminuzione delle tasse e l'apertura delle barriere doganali, avvenuta tra il 2003 e il 2005 in Danimarca, Svezia e Finlandia, inaspettatamente sono diminuiti sia i consumi alcolici (in Danimarca e In Svezia), sia i problemi alcol-correlati riferiti soggettivamente (ma non le diagnosi riportate nei registri sanitari, che sono cresciuti), (Bloomfield et al., 2010; Room et al., 2013).

Nell'ambito del progetto AMPHORA, co-finanziato dall'UE, sono state analizzate le correlazioni tra fattori contestuali e di politica alcologica, consumo alcolico e danni alcol-correlati tra il 1960 e il 2008 in 12 Paesi europei rappresentanti le regioni settentrionali (Norvegia Svezia, Finlandia), occidentali (Regno Unito, Paesi Bassi), orientali (Polonia, Ungheria), centrali (Austria, Svizzera), e meridionali (Francia, Spagna, Italia), tramite il metodo delle Serie temporali e quello delle Reti neuronali artificiali.

Si è così dimostrato che, se si considerano complessivamente i 12 paesi, le restrizioni al tasso alcolemico consentito durante la guida, alla disponibilità di vendita delle bevande alcoliche e all'età minima per il consumo risultano significativamente associate alla riduzione nei consumi, ma i risultati variano sostanzialmente da paese a paese.

Si sono persino paradossalmente osservate correlazioni positive tra aumento di consumi e introduzione dei limiti di età minimi in Austria, di restrizioni del tasso alcolemico consentito alla guida in Francia, Olanda e Spagna, e di restrizioni alla disponibilità di vendita in Regno Unito e in Svizzera (Allamani, Pepe e Voller, 2015).

Una meta-analisi sugli stessi dati ha confermato che l'associazione tra misure politiche e consumo totale di bevande alcoliche varia tra un paese e l'altro (Baccini \& Carreras, 2014).

Una revisione sistematica di cinquantacinque rilevanti pubblicazioni econometriche del periodo 1987-2013 non ha trovato coerenza di risultati sull'effetto dell'introduzione di tasse e limiti di importazione sui consumi alcolici nei confronti dei danni associati in Danimarca, Svezia, Finlandia, Svizzera e Hong Kong (Nelson \& McNall, 2015).

Gli stessi autori hanno riesaminato gli esperimenti naturali (cioè quei disegni di ricerca che valutano i cambiamenti avvenuti in una intera popolazione dopo che si sono realizzati gli interventi preventivi imposti alla stessa) pubblicati tra il 2003 e il 2015, che analizzavano i danni alcol-correlati conseguenti ai cambiamenti nelle politiche di tassazione, prezzo e disponibilità delle bevande alcoliche in nove paesi (Australia, Danimarca, Finlandia, Hong Kong, Islanda, Russia, Svezia, Svizzera e Stati Uniti) (Nelson e McNall, 2016).

Dai risultati si evince che la fiducia posta in queste misure è troppo alta, poiché esse hanno in realtà effetti selettivi secondo le diverse sottopopolazioni.

Per esempio, le modifiche delle tasse e dei prezzi hanno avuto effetti positivi solo sulla mortalità epatica degli anziani e solo in Finlandia e in Russia; per la maggior parte dei casi le poli- tiche alcologiche non hanno rivelato effetti di rilievo sulle aggressioni e sulla guida in stato di ebbrezza; e gli impatti sull'ubriachezza sono risultati variabili, nei paesi nordici, secondo la sotto-popolazione esaminata.

Un altro studio ha analizzato la relazione tra indici di severità delle politiche alcologiche messe in atto nel 2005 in 30 paesi OCSE, da un lato, e consumi alcolici totali e anni di vita persi aggiustati per disabilità (DALY), dall'altro.

Si è concluso che non si sono riscontrate correlazioni significative (Poikolainen, 2016).

\section{Come interpretare la incerta efficacia delle politiche alcologiche: limiti nella praticabi- lità degli studi basati sull'evidenza nel campo preventivo}

Come spiegare l'imprevedibilità dell'efficacia delle politiche alcologiche, di cui per molti sarebbe dimostrata l'evidenza scientifica?

Si tratta di limiti delle ricerche condotte, oppure non si considera a sufficienza la questione della difficile implementazione delle misure adottate nei diversi contesti?

Oppure, ancora, si ha a che fare con la grande variabilità dei fattori di paesi e culture e dunque con la complessità che necessariamente caratterizza l'azione preventiva rivolta ai cittadini?

Proviamo a fornire quattro ipotesi di risposta alle domande che ci siamo poste.

a) L'estendere l'evidenza scientifica di un progetto da un paese a un altro ha limiti intrinseci.

La letteratura sull'efficacia delle politiche in materia di alcol si riferisce spesso a studi empirici e a iniziative di prevenzione rivolte ad aree specifiche di un paese o a particolari campioni di popolazione (come gli studenti delle scuole primarie e secondarie), mentre sono poche le pubblicazioni su contesti allargati a un'intera nazione e alla sua popolazione (fra questi, Albalate, 2006; Pridemore et al., 2009, 2013; Zatonski et al., 2015).

Così l'assunto che studi condotti in contesti limitati possano valere per un'intera popolazione nazionale, può essere ingannevole.

Un altro problema si verifica quando l'evidenza dell'efficacia si basa su studi epidemiologici condotti in una cultura specifica (ad esempio in paesi tradizionalmente sensibili alle conseguenze del bere, cioè i paesi scandinavi e quelli anglofoni) ed è generalizzata, ovvero trasferita in paesi con ben diversa cultura del bere (ad esempio quelli dell'Europa mediterranea) (Room et al., 2005; Craplet, 2006; Anderson \& Baumberg, 2006; Peele, 2010; Berten et al., 2013).

Tutto ciò mentre gli organi internazionali da un lato affermano che le misure di politica alcologica devono essere adattate alle condizioni diverse di culture e paesi diversi (World Health Organization, 1993, 2015; UCL, 2013) e, dall'altro, raccomandano linee guida sul controllo del bere a rischio e del bere dannoso alla generalità dei paesi, incluse Asia, Africa e America meridionale (vedi Sornpaisarn et al., 2012; Casswell, 
2012; CNAPA, 2014; Ferreira-Borges et al., 2015; OECD, 2015; Pan American Organization, 2017).

L'impatto di tali misure preventive può rivelarsi deludente per il fatto stesso che sono applicate in culture diverse da quelle in cui sono state generate.

Il problema cruciale resta dunque quello della trasferibilità delle evidenze da un contesto all'altro.

b) I cambiamenti nel consumo di bevande alcoliche e nei danni correlati riflettono più $i$ cambiamenti sociali che le politiche preventive.

Le ricerche hanno confermato che i cambiamenti nel consumo di bevande alcoliche e nei danni associati sono soprattutto un riflesso dei cambiamenti della società nelle sue componenti demografiche, economiche, politiche, culturali e ideologiche, piuttosto che l'effetto dell'introduzione delle politiche preventive (Room, 1997; McLeod et al., 2016).

Secondo il progetto AMPHORA, le modifiche occorse tra gli anni 1960 e 2000 nel consumo alcolico globale dei 12 paesi europei studiati sono sempre avvenute in correlazione significativa con gli aumenti di urbanizzazione, reddito ed età delle madri al momento del parto, che sono considerati indicatori di miglioramento socioeconomico di una popolazione (Allamani, Pepe e Voller, 2015). Tuttavia questi fattori si associano in modo inverso secondo i paesi e, specificamente, hanno condotto a:

- un aumento del consumo alcolico totale e a un decremento nel consumo di superalcolici nel nord Europa;

- un aumento del consumo alcolico totale, di birra, e di vino nelle regioni centroeuropee;

- una riduzione del consumo alcolico totale, di vino e di superalcolici, con aumento del consumo di birra, nelle regioni sudeuropee.

È di particolare rilevanza che le modifiche nei consumi di bevande alcoliche si siano prodotte in alcuni casi senza la presenza e/o l'introduzione di misure politiche preventive nazionali, com'è avvenuto con la riduzione dei consumi iniziata in Francia negli anni '50 e in Spagna e in Italia negli anni '70, o con l'aumento dei medesimi in Finlandia e Norvegia negli anni '60.

In tutti questi casi, i cambiamenti nel consumo erano solo correlati ai fattori socio-economici e demografici (Allamani, Pepe e Voller, 2015; Allamani, Olimpi et al., 2014; si veda anche, per l'Italia, Cipriani \& Prina, 2007).

È stato anche dimostrato che un'età avanzata delle madri al momento del parto e l'invecchiamento della popolazione indicatori contestuali di un aumento del benessere - erano associati in modo significativo a una riduzione dei decessi per incidenti stradali in tutti i paesi e, solo nelle regioni dell'Europa meridionale, dei decessi dovuti a patologia epatica cronica (Allamani, Pepe e Voller, 2015).

I fattori contestuali si sono rivelati in grado di spiegare i cambiamenti nel consumo e nel prodursi di danni in grado maggiore delle misure preventive.

Infatti, mediante alcune misure di correlazione quadratica parziale, i fattori socio-economici e demografici spiegano il $71 \%$ dei cambiamenti dei consumi, mentre le politiche preventive ne spiegano il $38 \%$; i primi possono spiegare il $43 \%$ dei decessi dovuti a patologie epatiche e il $52 \%$ di quelli dovu- ti agli incidenti stradali, mentre le seconde spiegano rispettivamente il $30 \%$ e il $31 \%$ dei decessi (Allamani, Pepe e Voller, 2015).

Altri studi si sono concentrati sull'impatto che i grandi rivolgimenti socio-politici di una popolazione hanno sui modi di bere: ne sono esempi storici i processi di dislocazione e di conseguente alcolismo che hanno colpito i membri della comunità di Orkney in Scozia reclutati per lavorare in Canada nel XVIII secolo; o lo sviluppo di forme di dipendenza alcolica tra i popoli aborigeni nordamericani che erano stati trasferiti in contesti culturali a loro estranei con l'arrivo degli europei nel Nuovo Mondo - forme di dipendenza che, peraltro, in qualche caso sono regredite con la riacquisizione dei valori culturali originali (Alexander, 2008).

La percezione della normalità o dannosità delle bevande alcoliche per la salute è anch'esso un valore che si modifica nel tempo e che è in grado di influenzare il consumo, come hanno mostrano gli studi di Nordlund e Østhus (2013) e di Tusini (2006).

Si è anche osservato che un aumento della preoccupazione sulle conseguenze del bere in Danimarca tra il 2003 e il 2013 (Bloomfield et al., 2016) e in Australia del 1995 al 2010 (Callinan et al., 2014) si è accompagnato a una riduzione o stagnazione dei consumi alcolici.

\section{c) L'attuazione delle politiche alcologiche è determinata dalla variabile complessità delle azioni locali}

Ai fini della valutazione dell'impatto dei vari interventi è poi molto importante capire se le misure pianificate siano state implementate in maniera adeguata lungo tutti i punti della catena relativa agli attori coinvolti nel programma e se siano state accolte e messe in atto dai soggetti interessati della comunità.

Infatti nel campo delle politiche socio-sanitarie, numerosi sono i fattori implicati: l'approvazione di normative o atti amministrativi a livello dei governi locali, la loro attuazione da parte degli uffici e degli operatori responsabili, l'atteggiamento della cultura locale in ordine all'ottemperanza alle leggi, la disponibilità e prontezza della popolazione a partecipare e a recepire le proposte di carattere preventivo (Oetting et al., 2001; Prina, 2010; Karlsson et al., 2012; Nordlund \& Østhus, 2013; Allamani, Pepe et al., 2014,).

In genere, gli aspetti culturali sono rilevabili più a livello "meso" e "micro", ossia sul piano di distretto o comunità locale, che non a livello di nazione o di un'intera cultura (Beccaria \& Rolando, 2016).

In più, le azioni preventive implicano risvolti politici ed etici (Larsson, 1990).

Così si sono spesso notate relazioni contraddittorie tra esperti e cittadini, nonché tra studiosi della prevenzione e attivisti locali e politici originate da differenti concezioni sulla salute pubblica e sulle azioni locali da intraprendere, concezioni legate a diverse ideologie, mentalità, esperienze, interessi (Lindblath \& Hanson, 1993; Roumeliotis, 2016).

Sarebbe tuttavia necessario che nell'ambito dei progetti di prevenzione orientati alle comunità si costituisse un "sodalizio positivo" o un "rispettoso matrimonio" tra conoscenza scientifica e esperienza comunitaria, con gli esperti che si limitano alla identificazione degli interventi che possano avere effetto e 
i membri della comunità che si interessano ad adattare e attuare gli interventi pianificati (Holder \& Reynolds, 1999).

d) L'indebolimento del modello del consumo totale su cui sono basate le politiche alcologiche rende queste meno affidabili

Le moderne misure di politica alcologica sono fondate sulla teoria, o modello, del consumo totale, elaborato negli anni '70 da Kettil Bruun sulla base delle ricerche dell'epidemiologo francese Sully Ledermann, e subito divenuto il pilastro della strategia dell'Organizzazione Mondiale della Sanità (Ledermann, 1956; Bruun et al., 1975; World Health Organization, 1993; Anderson \& Baumberg, 2006).

In base a questo modello, la distribuzione del consumo alcolico è log-normale e vi è un legame funzionale tra il consumo alcolico medio di una popolazione e la prevalenza di forti bevitori, nonché la mortalità alcol-correlata (Ledermann, 1956; Leifman, 1996).

Il modello implica che i consumatori "normali" contribuiscano alla prevalenza dei forti bevitori e ai problemi relativi: se i primi riducono i loro consumi, la prevalenza dei secondi diminuirà.

E se i primi aumentano i loro consumi, i secondi aumenteranno (Skog, 1985).

Di conseguenza, la riduzione del livello di consumo di una popolazione, dopo che una politica preventiva vi è stata realizzata, è ritenuta un indicatore di successo della politica stessa.

Ciò non di meno, passando in rassegna le pubblicazioni più rilevanti realizzate tra il 2005 e il 2015, Norman Giesbrescht e colleghi hanno evidenziato come l'introduzione di diverse misure politiche abbia potuto produrre effetti sostanziali non sul consumo di tutta una popolazione, ma solo su quello di alcuni suoi sottogruppi - giovani, donne, forti bevitori (Giesbrecht et al., 2016).

Altri autori hanno sottolineato che le curve di consumo a rischio dipendono anzitutto dalle disuguaglianze sociali (Jones e coll., 2015; UNICEF, 2016).

Simulando l'effetto dell'introduzione del prezzo minimo per unità alcolica sulla popolazione inglese, il gruppo di Sheffield ha chiarito che la risultante riduzione del consumo e del danno avverrebbe solo per i sottogruppi dei forti bevitori, appartenenti alle fasce socioeconomiche più svantaggiate (Holmes et al., 2014; Meier et al., 2016).

Ci sarebbe poi da notare che il modello sviluppato da Kettil Bruun considera solo il consumo alcolico totale, senza tener conto dei differenti modi di consumo delle bevande, inclusa la rapidità della loro ingestione, che è un segno distintivo dei forti bevitori e degli abusatori occasionali (Poikolainen, 2017).

Altri studiosi hanno osservato che l'impatto delle politiche alcologiche sui danni correlati al bere è spesso maggiore rispetto all'impatto che esse hanno sul consumo alcolico (Room, 2004; Jiang e Room, 2016).

Il punto è sostenuto anche dal progetto AMPHORA, che ha rilevato come nei paesi europei studiati le misure alcologiche hanno avuto un effetto diretto sulla mortalità alcol correlata più grande rispetto all'effetto mediato dal consumo di bevande alcoliche sulla mortalità stessa (AMPHORA, 2013).

Kim Bloomfield e colleghi hanno poi notato in Danimarca, tra il 2003 e il 2013, trend divergenti tra consumo alcolico, in riduzione, e danno alcol-correlato, in crescita, in corrispondenza di un insieme di politiche che ha aumentato la disponibilità di bevande alcoliche e ristretto la loro accessibilità ai minori, con tassazione e prezzi stabili o ridotti (Bloomfield et al., 2016).

Ne consegue che le misure preventive, più che dare attenzione al consumo di bevande alcoliche di un'intera popolazione, mostrerebbero più frutti concentrandosi su sottogruppi di popolazione e sulla riduzione dei loro consumi e danni associati (Poikolainen, 2017).

Addirittura Kevin Shield e colleghi suggeriscono alle istituzioni di salute pubblica di abbandonare le raccomandazioni proprie delle linee guida per il bere a rischio, e di prestare piuttosto attenzione ai più concreti rischi di mortalità (Shield et al., 2016).

\section{Altre ipotesi per spiegare la variabile effica- cia delle politiche alcologiche: limiti della ricerca in alcologia}

Il riscontro di variabilità nell' efficacia delle misure preventive può essere attribuito, come detto più sopra, alla difficile generalizzabilità degli studi alcologici.

Un aspetto correlato a tale difficoltà riguarda la scarsa replicabilità delle ricerche.

In linea di massima, solo una minima parte della ricerca scientifica pubblicata in campo biomedico e psicologico (secondo alcune stime il 25\%) è riproducibile, e tale osservazione si può estendere anche al campo dell'alcologia.

Le ragioni sono state identificate nella ridotta ampiezza dei campioni, nella bassa dimensione dell'effetto, nell'analisi a posteriori (senza ipotesi precedenti l'indagine), nel confitto d'interessi e nell'isolamento tra i gruppi di ricerca (Ioannidis, 2005; Room, 2016; Munafò, 2017).

L'incertezza dei risultati delle misure preventive potrebbe essere riferibile, a sua volta, a una corrispondente incertezza, o ambiguità, dei principi e delle procedure su cui si basa anche la ricerca epidemiologica sull'alcol.

Quest'ambiguità si riflette nei concetti e nei processi di rischio e causalità, nella definizione della raccolta dei dati, nella valutazione delle azioni politiche.

La definizione dei concetti e dei processi per stabilire il rischio e il margine di esposizione alle sostanze oggetto dell'indagine, e i relativi criteri di misurazione, non è stabile ma tende a cambiare nel tempo, il che può esser dovuto all'importanza che il consenso e le convenzioni tra gli scienziati, più che l'elaborazione di un'informazione oggettiva, hanno a proposito; ad esempio è sorprendente che la determinazione dei rischi relativi al bere sia stata fatta sulla base di "ciò che il pubblico sembra aver voglia di accettare" (Starr, 1969; Rehm et al., 2014; Antunes e Gonzalez, 2015; ALICE RAP, 2015).

Gli studiosi non hanno una posizione concorde nel definire la relazione tra la bevanda alcolica bevuta e i problemi ad essa attributi, ma la qualificazione di tale relazione, nonché i criteri per determinarla, variano secondo gli autori: da causale, a multi-causale, a multifattoriale, a probabile (Hill, 1965; Feller, 1968; Rothman et al., 2008; Connor, 2016; Shield, Parkin et al., 2016). 
Secondo la qualificazione scelta, nell'oscillazione tra gli estremi dell'interpretazione di causalità e di quella di probabilità, differiranno di conseguenza i significati e la forza che acquistano i nessi tra il bere e i problemi ad esso associati.

Poiché tale incertezza, specie quando le informazioni devono essere trasmesse a livello di sanità pubblica, può divenire preoccupante e controproducente, qualche autore ha concluso che la forza dell'evidenza per cui un'azione preventiva va attuata è da porre nell' ambito delle scelte etiche, piuttosto che scientifiche (Narduzzi et al., 2017).

In questa prospettiva, le decisioni relative al tipo d'intervento da pianificare dovrebbero rispondere a domande pratiche preliminari, del tipo: «chi avrebbe beneficiato e chi sarebbe stato danneggiato, nel caso che l'evidenza si rivelasse incerta o i risultati risultassero erronei?».

Inoltre la determinazione dei consumi reali riferiti dagli intervistati nelle inchieste epidemiologiche appare minato dalla progressiva contrazione nel tasso di risposta ai questionari, che in Europa è spesso sotto il $50 \%$, nonché dalla incertezza dovuta alla variabilità del volume contenuto nei bicchieri, oggetto delle domande degli intervistatori (Moskalewicz, 2012; Kerr et al., 2009; Zupan et al., 2017).

Oltre a ciò, la stragrande maggioranza delle ricerche alcologiche si concentra sul rischio di bere e sui danni attribuibili all'alcol, con gli interventi di salute pubblica diretti alla restrizione o alla soppressione dei comportamenti orientati al consumo.

Viene così trascurata l'esperienza di piacere e di benessere individuale e sociale degli intervistati che, se raccolta, potrebbe fornire utili indicazioni per la migliore comprensione della salute globale e della sua percezione (Peele e Grant, 1999; ALICE RAP, 2016).

Infine, sappiamo ancora troppo poco sugli impatti che hanno avuto le politiche alcologiche nazionali, e la mancanza di valutazioni e di resoconti non contribuisce allo sviluppo di nuovi programmi preventivi (Moskalewicz, 2012; Anderson, 2012). La riluttanza dei ricercatori e dei decisori politici nel produrre valutazione può dipendere dagli interessi e dalle ideologie in gioco, che rendono difficile accettare possibili insuccessi (Einstein, 2012).

Un altro motivo è che i cambiamenti a livello di comunità sono spesso difficili da misurare, richiedendo più tempo e fondi di quanto si possano permettere politici e ricercatori (Holder e Moore, 2000; Moskalewicz, 2012).

\section{Concludendo}

L'articolo ha mostrato i limiti delle posizioni che sostengono l'opportunità di politiche alcologiche indifferenziate e solamente orientate al controllo dei consumi di bevande alcoliche. Si è messo in evidenza che le politiche non possono essere applicate ugualmente in tutti $\mathrm{i}$ paesi, in quanto risultano non ugualmente efficaci, e a volte inefficaci, secondo i paesi e i sottogruppi di popolazione, a causa dei differenti contesti sociali, demografici, culturali e economici.

Inoltre, alcuni limiti della ricerca epidemiologica, e lo scarso ricorso ai processi di valutazione, rendono i risultati degli interventi preventivi incerti, riducendo la generalizzabilità degli esiti e suggerendo un approccio precauzionale nella creazione e diffusione dei messaggi di salute pubblica.

Tenuto conto di queste limitazioni, e ai fini di superarle, quando è previsto un programma di prevenzione delle bevande alcoliche basato sulle evidenze in un paese o regione, gli esperti, i responsabili politici e le parti interessate dovrebbero almeno considerare l'importanza decisiva di:

- nella fase di pianificazione dell'introduzione di una misura politica, raccogliere informazioni specifiche sui fattori sociali, economici e demografici specifici del proprio paese/comunità;

- prevedere interventi selettivi su sottogruppi di popolazione, come giovani, donne, anziani, forti bevitori (Mäkelä et al., 2002; Ahlström, 2008; CNAPA, 2014);

- tener conto dei danni associati al bere - come cirrosi epatica, incidenti stradali, cancro - oltre che del consumo alcolico e delle modalità del bere;

- pianificare un programma di valutazione, in modo da poter replicare interventi di successo e rivedere quelli che sono falliti.

Si deve aggiungere che, in particolare in Europa, le sfide rappresentate dalle crescenti disuguaglianze nella disponibilità $\mathrm{e}$ nell'accesso alle risorse di base e dall'aumentare delle popolazioni di migranti, avranno impatto anche sugli stili di vita e sui modi di bere della popolazione nel suo complesso (World Health Organization, 2014 b).

Occorre dunque agire con urgenza nel campo della ricerca preventiva e, allo stesso tempo, considerare nuove tipologie di politiche di intervento efficaci.

Tra queste, è da considerare l'uso dei metodi predittivi, che si propongono di prevedere l'efficacia delle politiche preventive volte a ridurre $\mathrm{i}$ danni alcol-correlati e migliorare la qualità della vita nel breve e medio termine (Buscema, 1995; Oderkirk et al., 2012).

\section{Bibliografia}

Albalate D. (2006). Lowering blood alcohol content levels to save lives: the European experience. Barcelona, Research Institute of Applied Economics (No. CREAP20006-07). www.ub.edu/irea/ working_papers/2006/200603.pdf.

Ahlström S. (2008). Alcohol use and problems among older women and men: A review. Nordic Studies on Alcohol and Drugs, 25(2): 154-161.

Alexander B.K. (2008). The globalisation of addiction: A study in poverty of the spirit. Oxford: Oxford University Press.

ALICE RAP (2015). Position paper sul margine di esposizione (per il Parlamento Europeo) www.alicerap.eu/resources/documents/ cat_view/1-alice-rap-project-documents/19-policy-paperseries.html.

ALICE RAP (2016). Addiction and Lifestyles in Contemporary Europe - Reframing Addictions Project Well Being Library www.alicerap.eu/resources/documents/cat_view/18-well-beinglibrary.html (accessed July 2016).

Allamani A., Pepe P., Voller F. (2015). L'Europa tra culture del bere e politiche di controllo alcologico. Newsletter "Clinica dell'Alcolismo", V(20): I-VIII - Mission, 44: 31-38.

Allamani A., Olimpi N., Pepe P., Cipriani F.(2014). Trends in Consumption of Alcoholic Beverages and Policy Interventions in Europe: An Uncertainty "Associated" Perspective. Substance Use and Misuse, 49(12): 1531-1545. 
Allamani A., Pepe P., Baccini M., Massini G., Voller F. (2014). Europe. An analysis of changes in the consumption of alcoholic beverages: the interaction between consumption, related harms, contextual factors and alcoholic beverage control policies. Substance Use and Misuse, 49(12): 1692-1715.

AMPHORA Workpackage3 (2013). Report of an analysis of European alcohol-related cultural, social and policy interactions and their impact on alcohol consumption and alcohol-related harm http://amphoraproject.net/w2box/data/Deliverables/ AMPHORA_WP3_D3.2.pdf.

Anderson P., Chisholm D., Fuhr D. (2009). Effectiveness and costeffectiveness of policies and programmes to reduce the harm caused by alcohol. Lancet, 373: 2234-46.

Anderson P., Møller L., Galea G. (2012). Alcohol in the European Union. Consumption, harm and policy approaches. World Health Organisation, Regional Office for Europe, Copenhagen.

Anderson P., Baumberg B. (2006), Stakeholders' views of alcohol policy. Nordic Studies on Alcohol and Drugs, 23: 393-414.

Anderson P., Baumberg B. (2006). Alcohol in Europe. A public health perspective. London: Institute of Alcohol Studies (http://ec.europa.eu/health-eu/doc/alcoholineu_content_en.pdf).

Angus C., Latimer N., Preston L., Li J., Purshouse R. (2014). What are the implications for policy makers? A systematic review of the cost-effectiveness of screening and brief interventions for alcohol misuse in primary care. Frontiers in Psychiatry, 5: 114.

Antunes R., Gonzalez V. (2015). A Production Model for Construction: A Theoretical Framework. Buildings, 5(1): 209228. doi: 10.3390/buildings5010209.

Babor T.F., Caetano R., Casswell S., Edwards G., Giesbrecht N., Graham K., Grube J.W., Hill L., Holder H., Homel R., Livingston M., Österberg E., Rehm J., Room R., and Rossow I. (2010). Alcohol: no ordinary commodity, Research and public policy, 2nd ed. Oxford: Oxford University Press.

Babor T.F., Del Boca F., Bray J.W. (2017). Screening, Brief Intervention and Referral to Treatment: implications of SAMHSA's SBIRT initiative for substance abuse policy and practice. Addiction, 112, S2: 110-117.

Baccini M., Carreras G. (2014). Analyzing and comparing the association between Control Policy Measures and Alcohol Consumption in Europe. Substance Use \& Misuse, 49: 1684-1691.

Beccaria F., Rolando S. (2016). The more you know, the better you drink: The new drinking culture in Italian wine production areas. Drugs: Education, Prevention and Policy, 1-9 (early on line). doi: 10.3109/09687637.2016.1149149.

Beccaria F., Rolando S., Ascani P. (2012). Alcohol consumption and Quality of Life among young adults. A comparison among three European countries. Substance Use and Misuse, 47(11): 1214-23.

Berten H., Vetternburg, N. Steketee M., Steketee M., Jonkman H. van den Toorn J. et al. (2013). Policies, programmes and interventions: results of focus groups with practionners, policymakers and researchers. In: Steketee M., Jonkman H., Berten H., Vettenburg N. (eds.). Alcohol use Among Adolescents in Europe Environmental Research and Preventive Actions. Utrecht - anche in www.aaaprevent.eu/, pp. 314-317.

Buscema P.M. (1995). Squashing Theory: a Prediction Approach for Drug Behavior. Drug and Society, 8(3-4): 103-109.

Buscema P.M., Massini G., Maurelli G. (2014). Artificial Neural Networks: An overview. Substance Use and Misuse, 49: 155-1568.

Bloomfield K., Wicki M., Gustafsson N-K., Mäkelä P., Room R. (2010). Changes in Alcohol-Related Problems after Alcohol Policy Changes in Denmark, Finland, and Sweden. Journal of Studies on Alcohol and Drugs, 71(1): 32-40.

Bloomfield K., Karlsson T., Grittner U. (2016). How do drinking cultures change? - or do they? A provisional model based on evidence of transitions in Denmark. Drugs: education, prevention and policy, 23(4): 283-92.

Bruun K., Edwards G., Lumio M., Mäkelä K., Pan L., Popham R.E., Room R., Schmidt W., Skog, O.J., Sulkunen P., Österberg E. (1975). Alcohol control policies in public health perspective. The Finnish Foundation for Alcohol Studies, Volume 25, Forssa.

Callinan S., Room R.. Livingston M. (2014). Changes in Australian attitudes to alcohol policy: 1995-2010. Drug and Alcohol Review, 33: 227-234.

Casswell S. (2012). Current status of alcohol marketing policy - an urgent challenge for global governance. Addiction, 107: 478-485. doi: 10.1111/j.1360-0443.2011.03701.x.

Cipriani F., Prina F. (2007). The Research Outcome: Summary and Conclusions on the Reduction in Wine Consumption in Italy. In: Beccaria F., Allamani A. (a cura di). Changes in the Consumption of Alcoholic Beverages in Italy: Studies of the Decrease in Consumption between the $1970 \mathrm{~s}$ and the $2000 \mathrm{~s}$. Contemporary Drug Problems, 34(2): 361-378.

Connor J. (2016). Alcohol consumption as a cause of cancer. Addiction. doi: 10.1111/add.13477.

CNAPA Committee on National Alcohol Policy and Action Action Plan (2014). Youth Drinking and on Heavy Episodic Drinking (Binge Drinking) (2014-2016), 16 Settembre, http://ec.europa.eu/ health/alcohol/docs/2014_2016_actionplan_youthdrinking_en.pdf.

Craplet M. (2006). Open letter to my friends and colleagues. Addiction, 101: 450-452.

de Bruijn A., Johansen I., van den Broeck A. (2010). Effective alcohol marketing regulations: a proposed framework to evaluate existing alcohol marketing regulations. Utrecht: Dutch Institute for Alcohol Policy.

de Bruijn A., Tanghe J., de Leeuw R., Engels R., Anderson P., Beccaria F., Bujalski M., Celata C., Gosselt J., Schreckenberg D., Słodownik L., Wothge J., van Dalen W. (2016). European longitudinal study on the relationship between adolescents' alcohol marketing exposure and alcohol use. Addiction. doi: $10.1111 /$ add.13455.

Einstein S. (2012). Introduction. Substance Use and Misuse, 47: 1360-1364.

Elder R.W., Lawrence B., Ferguson A., Naimi T.S., Brewer R.D. Chattopadhyay S.K., Toomey T.L., Fielding J.E., the Task Force on Community Preventive Services (2010). The Effectiveness of Tax Policy Interventions for Reducing Excessive Alcohol Consumption and Related Harms. American Journal of Preventive Medicine, 38(2): 217-229.

European Health for All Database (HFA-DB), World Health Organization (2016). http://data.euro.who.int/hfadb/.

Commissione Europea (2010). Direzione Generale Salute e Consumatori - Development of alcohol policy and action in EU Member States 2006-2009. Allegato 1 al primo Progress Report sull'implementazione della strategia dell'UE in materia di alcol. Commissione europea al link: http://ec.europa.eu/health/ ph_determinants/life_style/alcohol/documents/alcohol_ progress.pdf.

Feller W. (1968). An Introduction to Probability Theory and Its Applications, (Vol. 1), 3rd Ed. Taiwan: Wiley and Sons.

Ferreira-Borges C., Esser M.B., Dias S. et al. (2015). Alcohol Control Policies in 46 African Countries: Opportunities for Improvement. Alcohol Alcohol, 50: 470-6.

Foxcroft D.R., Tsertsvadze A. (2011). Universal multi-component prevention programs for alcohol misuse in young people. Cochrane Database of Systematic Reviews, (9): CD009307.

Foxcroft D.R., Coombes L., Wood S., Allen D., Almeida Santimano N.M.L. (2014). Motivational interviewing for alcohol misuse in 
young adults. Cochrane Database of Systematic Reviews, (8): CD007025.

Grant S., Pedersen E.R., Osilla K.C., Kulesza M., D'Amico E.J. (2016). Reviewing and interpreting the effects of brief alcohol interventions: comment on a Cochrane review about motivational interviewing for young adults. Addiction, 111(9): 1521-1527.

Giesbrecht N., Wettlaufer A., Cukier S., Geddie G., Gonçalves A.H., Reisdorfer E. (2016). Do alcohol pricing and availability have differential effects on subpopulations? A commentary. International Journal of Alcohol and Drug Research, 5(3): 89-99.

Green C.P., Heywood J.S., Navarro M. (2014). Did liberalising bar hours decrease traffic accidents? Journal of Health Economics, 35: 189-198. doi: 10.1016/j.jhealeco.2014.03.007.

Gruenewald P.J. (2011). Regulating availability: how access to alcohol affects drinking and problems in youth and adults. Alcohol Research \& Health, 34(2): 248-256.

Hahn R.A., Kuzara J.L., Elder R., Brewer R., Chattopadhyay S., Fielding J., Naimi T.S., Toomey T., Middleton J.C., Lawrence B. \& Task Force on Community Preventive Service (2010). Effectiveness of policies restricting hours of alcohol sales in preventing excessive alcohol consumption and related harms. American Journal of Preventive Medicine, 39(6): 590-604.

Heath D. (ed.) (1995). International handbook on alcohol and culture. Westport, CN: Greenwood Press.

Hill A.B. (1965). The environment and disease: association or causation? Proceedings of the Royal Society of Medicine, 58: 295300.

Ioannidis J.P.A. (2005). Why most published research findings are false. PLoS Med, 2(8): el24.

Holder D.H., Reynolds R. (1999). Alcohol policy and community action: Experiences from a national prevention trial to reduce alcohol problems. Community action to prevent alcohol problems. Copenhagen, WHO Regional Office for Europe, pp. 78-89. www.euro.who.int/__data/assets/pdf_file/0003/119181/E63694. pdf.

Holder H.D., Moore R.S. (2000). Institutionalization of Community Action Projects to Reduce Alcohol-Use Related Problems: Systematic Facilitators. Substance Use and Misuse, 35(1, 2): 7586.

Holmes J., Meng Y., Meier PS., Brennan A., Angus C., CampbellBurton A., Guo Y., Hill-McManus D., Purshouse R.C. (2014). Effects of minimum unit pricing for alcohol on different income and socioeconomic groups: a modelling study. The Lancet, 383(9929): 1655-64.

Jiang H., Room R. (2016). The many facets of alcohol policy. International Journal of Alcohol and Drug Research, 5(3): 85-87.

Järvinen M., Room R. (eds.) (2007). Youth drinking cultures. European experiences. Aldershot: Ashgate.

Jones L., Bates G., McCoy E. et al. (2015). Relationship between alcohol-attributable disease and socioeconomic status, and the role of alcohol consumption in this relationship: a systematic review and meta-analysis. BMC Public Health, 5: 400. doi: 10.1186/s12889-015-1720-7.

Kerr W.C., Patterson D., Koenen M.A., Greenfield T.K. (2009). Large drinks are no mistake: Glass size, but not shape, affects alcoholic beverage drink pours. Drug and Alcohol Review, 28(4): 360-365. doi: 10.1111/j.1465-3362.2009.00056.x.

Karlsson T., Lindemann M., Österberg E. (2012). Does alcohol policy make any difference? Scales and consumption. In: Anderson P., Braddick F., Reynolds J., Gual A. (eds.). Alcohol policy in Europe: Evidence from AMPHORA, 2nd ed. The AMPHORA project, pp. 15-23. From: www.amphoraproject.net.

Kolosnitsyna M., Sitdikov M., Khorkina N. (2014) Availability restrictions and alcohol consumption: A case of restricted hours of alcohol sales in Russian regions. International Journal of Alcohol and Drug Research, 3(3): 193-201.

Kypri K., Craig Jones C., McElduff P., Barker D. (2011). Effects of restricting pub closing times on night-time assaults in an Australian city. Addiction, 106(2): 303-310.

Larsson S. (1990). Democracy and Community Action Programs. In: Research, Action and the Community: Experiences in the Prevention of Alcohol and other Drug Problems. OSAP Prevention Monograph-4. U.S. Department of Health and Human Services, Rockville, Maryland: 41-44.

Ledermann S. (1956). Alcool, alcoolisme, alcoolisation. Donnés scientifiques de caractére physiologique, économique et social. Presses Universitaires de France.

Leifman H. (1996). Perspectives on the aggregated consumption of alcohol. Summary article in Leifman H. Perspectives on alcohol prevention, Acta Universitatis Stockholmiensis, Stockholm studies in sociology, N.S. 3. Almqvist \& Wicksell International, Stockholm, pp. 3-9.

Lindbladh E., Hanson B.S. (1993). A critical analysis of different leadership approaches to community health work in Kirserberg, Sweden. Health Promotion International, 8(4): 291-297.

Lippy C., DeGue S. (2014). Exploring alcohol policy approaches to prevent sexual violence perpetration. Trauma, Violence \& Abuse, 17(1): 26-42.

Lolli G., Serianni E., Golder G.M., Luzzatto-Fegiz P. (1959). Alcohol in Italian culture: food and wine in relation to sobriety among Italians and Italian Americans. Glencoe, ILL: Free Press.

Mäkelä P., Rossow I., Tryggvesson K. (2002). Who drinks more or less when policies change? The evidence from 50 years of Nordic studies. In: Room R. (ed.). The effects of Nordic alcohol policies. What happens to drinking and harm when alcohol controls change? Helsinki, Finland: Nordic Council for Alcohol and Drug Research.

McCartt A.T., Hellinga L.A., Kirley B.B. (2010). The effects of minimum legal drinking age 21 laws on alcohol-related driving in the United States. Journal of Safety Research, 41(2): 173-181.

McLeod J., Gell L., Holmes J., Allamani A., Baumberg B., Bjerge B. et al. (2016). Determinants of transition form harmful to low-risk substance use and gambling. In: Gell L., Bühringer G., McLeod J., Forberger S., Holmes J., Lingford-Hughes A., Meier P. (eds.). What determines harm from addictive substances and behaviours? Oxford: Oxford University Press, pp. 114-155.

Meier PS., Holmes J., Angus C., Ally AK., Meng Y., Brennan A. (2016). Estimated effects of different alcohol taxation and price policies on health inequalities: A mathematical modelling study. PLOS Medicine, 13(2): e1001963.

Moskalewicz J. (2012). Common evidence base and monitoring. In: Anderson P., Møller P., Galea G. (2012). Alcohol in the European Union. World Health Organization, Regional Office for Europe, Copenhagen, pp. 111-117.

Møller L., Anderson P. (2012). Summary. In: Anderson P., Møller L., Galea G. Alcohol in the European Union. Consumption, harm and policy approaches. World Health Organisation, Regional Office for Europe, Copenhagen, pp 1-4.

Monteiro M.G., Babor T.E., Jernigan D., Brookes C. (2017). Alcohol marketing regulation: from research to public policy. Addiction, 112(Suppl. 1): 3-6.

Munafò M. (2017). Promoting reproducibility in addiction research. Addiction, 112: 1519-1520.

Nelson J. P. (2010). Alcohol advertising bans, consumption, and control policies in seventeen OECD countries, 1975-2000. Applied Economics, 42: 803-823.

Nelson J.P., McNall A. (2015). What Happens to Drinking and Harms When Alcohol Policy Changes? A Systematic Review of 
Five Natural Experiments for Alcohol Taxes, Prices, and Availability. Social Science Research Network, http://ssrn.com/ abstract=2612580 or http://dx.doi.org/10.2139/ssrn.2612580.

Nelson J.P., McNall A. (2016). Alcohol prices, taxes, and alcoholrelated harms: A critical review of natural experiments in alcohol policy for nine countries. Health Policy, 120(3): 264-272. doi: 10.1016/j.healthpol.2016.01.018.

Nordlund S., Østhus S. (2013). What is alcohol abuse? Attitudes to drinking in seven European Countries. Addiction Research and Theory, 21(5): 402-409.

Oderkirk J., Sassi F., Cecchini M., Astolfi R. (2012). Toward a New Comprehensive International Health and Health Care Policy Decision Support Tool. OECD Health Division, http://ec.europa.eu/health/projects/docs/2010_health_healthe are_policy_fr_en.pdf.

OECD (2015). Tackling Harmful Alcohol Use: Economics and Public Health Policy, oEcd publishing. doi: 10.1787/9789264181069-en.

Oetting E.R., Jumper-Thurman P., Plested B., Edwards R.W. (2001). Community Readiness and Health Services. Substance Use and Misuse, 36(6-7): 825-843.

Pan American Health Organization (2017). Technical Note: Background on Alcohol Marketing Regulation and Monitoring for the Protection of Public Health. Washington, D.C: PAHO, http://iris.paho.org/xmlui/bitstream/handle/123456789/33972/PA HONMH17003-eng.pdf? sequence $=1$ \&is Allowed $=\mathbf{y}$.

Patra J., Giesbrecht N., Rehm J., Bekmuradov D., Popova S. (2012). Are alcohol prices and taxes an evidence-based approach to reducing alcohol-related harm and promoting public health and safety? A Literature Review. Contemporary Drug Problems, 39: 7-48.

Peele S. (2010). Alcohol as evil - Temperance and policy. Addiction Research and Theory. 18(4): 374-382.

Peele S., Grant M. (eds.) (1999). Alcohol and Pleasure. A health perspective. ICAP, Taylor and Francis, United kingdom.

Pridemore W.A., Snowden A.J. (2009). Reduction in suicide mortality following a New National Alcohol Policy in Slovena: An interrupted time-series analysis. American Journal of Public Health, 99(5): 915-920.

Pridemore W.A., Chamlin M.V., Andreev E. (2013). Reduction in male suicide mortality following the 2006 Russian alcohol policy: An interrupted time series analysis. American Journal of Public Health, 103(11): 2021-2026.

Prina F. (2010). Le norme sulle bevande alcoliche e la loro applicazione: un bilancio nella prospettiva socio-giuridica. In: I giovani e l'alcool: consumi, abusi, politiche. Una rassegna critica multidisciplinare (in coll. con E. Tempesta), numero monografico di Salute e Società, IX(Supplemento al n. 3): 96-116.

Rehm J., Scafato E. (2011). Indicators of alcohol consumption and attributable harm for monitoring and surveillance in European Union countries. Addiction, 106: 4-10.

Rehm J., Lachenmeier D.W., Room R. (2014). Why does society accept higher risk for alcohol than for other voluntary or involuntary risks? BMC Medicine, 12: 189. www.biomedcentral.com/17417015/12/189.

Rehm J., Gmel G., Probst C., Shield K.D. (2015). RAHRA: Lifetimerisk of alcohol-attributable mortality based on different levels of alcohol consumption in seven European countries. Implications for low-risk drinking guidelines. Toronto, On, Canada: Centre for Addiction and Mental Health.

Room R. (1997). Alcohol, the individual and society: what history teaches us. Addiction, 92: S7-S11.

Room R., Babor T., Rehm J. (2005). Alcohol and public health. Lancet, 365: 519-30.
Room R. (2016). Sources of funding as an influence on alcohol studies. The International Journal Of Alcohol And Drug Research, 5(1): 15-16. doi: 10.7895/ijadr.v5il.231.

Roumeliotis F. (2016). Empowered Communities: Science, Ideology and the limits of Political Action. Nordic Studies on Alcohol and Drugs, 33: 343-359.

Schütze M., Boeing H., Pischon T., Rehm J., Kehoe T., Gmel G. et al. (2011). Alcohol-attributable burden of incidence of cancer in eight European countries, based on results from prospective cohort study. British Medical Journal, 342: d1584. www.bmj.com/content/342/bmj.d1584.

Shield K.D., Parkin D.M., Whiteman D.C., Rehm J., Viallon V., Marant Micakkef C., Vineis P., Rushton L., Bray F., Soerjomataram I. (2106). Population Attributable and Preventable Fractions: Cancer Risk Factor Surveillance, and Cancer Policy Projection. Current Epidemiology Reports, 3(3): 201-2113. doi: 10.1007/s40471-016-0085.

Shield K.D., Rylett M., Rehm J. (2016). Public health successes and missed opportunities. Trends in alcohol consumption and attributable mortality in the WHO European Region, 1990-2014. World Health Organization, Regional Office for Europe, Copenhagen.

Shults R.A., Elder R.W., Sleet D.A., Nichols L.J., Alao M.O., Carande-Kuli V.G., Zaza S., Sosi D.M., Thompson R.S., Task Force on Community Preventive Services (2001). Reviews of evidence regarding interventions to reduce alcohol-impaired driving. Americ an Journal of Preventive Medicine, 21: 66-88.

Skog O.J. (1985). The Collectivity of Drinking Cultures: A Theory of the Distribution of Alcohol Consumption. British Journal of Addiction, 80: 83-99.

Sornpaisarn B., Shield K.D., Rehm J. (2012). Alcohol taxation policy in Thailand: implications for other low- to middle-income countries. Addiction, 107: 1372-84.

Starr C. (1969). Social benefit versus technological risk. Science, 165: 1232-8.

Stockwell T.R., Chikritzhs T.N. (2009). Do relaxed trading hours for bars and clubs mean more relaxed drinking? A review of international research on the impacts of changes to permitted hours of drinking. Crime Prevention and Community Safety, 11(3): 153-171.

Sulkunen P. (1989). Drinking in France 1965-1979. An analysis of household consumption data. British Journal of Addiction, 84: 61-72.

Touvier M., Druesne-Pecollo N., Kesse-Guyot N., Andreeva V.A., Galan P., Hercberg S., Latino-Martel P. (2014). Demographic, socioeconomic, disease history, dietary and lifestyle cancer risk factors associated with alcohol consumption. International Journal of Cancer, 134: 445-459.

Treno A.J., Gruenewald P.J., Lee J.P., Rmeer L.G. (2007). The Sacramento Neighborhood Alcohol Prevention Project: outcomes from a community prevention trial. Journal of Studies on Alcohol and Drugs, 68(2): 197-207.

Tusini S. (2006). Le altre ipotesi interpretative sul calo del cosnumo di bevande alcoliche. In: Allamani A., Cipriani F., Prina F. (a cura di) (2006). I cambiamenti nei consumi di bevande alcoliche in Italia: uno studio esplorativo sul decremento dei consumi negli anni 1970-2000. I Quaderni dell'Osservatorio Permanente Giovani e Alcool n. 17. Roma: Casa Editrice Litos, pp. 117-170.

UCL-Institute of Health Equity (2013). Review of social determinants and the health divide in the WHO European Region: final report. World Health Organization (update reprint 2014), p. 158.

UNICEF Office of Research (2016). Fairness for Children: A League Table of Inequality in Child Well-Being in Rich Countries, 
Innocenti Report Card 13, Florence. www.unicef-irc.org/ publications/pdf/RC13_eng.pdf.

Wagenaar A.C., Salois M.J., Komro K.A. (2009). Effects of beverage alcohol price and tax levels on drinking: a meta-analysis of 1003 estimates from 112 studies. Addiction, 104: 179-190. doi: 10.1111/j.1360-0443.2008.02438.x.

Wicki M., Gmel G. (2011). Hospital admission rates for alcoholic intoxication after policy changes in the canton of Geneva, Switzerland. Drug and Alcohol Dependence, 118(2-3): 209-215.

Wilkinson C., Room R. (2009). Informational and warning labels on alcohol containers, sales, places and advertisements: experience internationally and evidence on effects. Drug and Alcohol Review, 28(4): 341-461.

World Health Organization (1993). European Alcohol Action Plan. Copenhagen, WHO, Regional Office for Europe.

World Health Organization (2011a). Global Information System on Alcohol and Health (GISAH) [database online]. Ginevra: WHO. www.who.int/gho/alcohol/en/.

World Health Organization (2011b). European action plan to reduce the harmful use of alcohol 2012-2020. Copenhagen, WHO, Regional Office for Europe. www.euro.who.int/_data/assets/ pdf_file/0008/178163/E96726.pdf.
World Health Organization (2014b). Alcohol and inequities. WHO Regional Office for Europe

World Health Organization (2014a) Global status report on alcohol and health. Geneva: World Health Organization. Copenhagen, Danimarca._www.euro.who.int/_data/assets/pdf_file/0003/ 247629/Alcohol-and-Inequities.pdf.

World Health Organization (2015). Beyond bias: exploring the cultural contexts of health and well-being measurement. Primo incontro del gruppo di esperti. WHO, Regional Office for Europe Copenaghen, Denmkark, Jan. 2015._www.euro.who.int/_data/ assets/pdf_file/0008/284903/Cultural-contexts-health.pdf.

Xuan Z., Blanchette J., Nelson T.F., Heeren T., Oussayef N., Naimi T.S. (2015). The alcohol policy environment and policy subgroups as predictors of binge drinking measures among US adults. American Journal of Public Health, 105(4): 816-822.

Zatoński W.A., Sulkowska U., Zatoński M.Z., Herbeç A.A. Muszyńska M.M. (2015). Alcohol taxation and premature mortality in Europe. Lancet, 385(9974): 1181. doi: 10.1016/S0140-6736(15)60634-1.

Zupan Z., Pechey R., Couturier D.L., Hollands G.J., Marteau T.M. (2017). Micro-drinking behaviours and consumption of wine in different wine glass sizes: a laboratory study. BMC Psychology, 5: 17. doi: 10.1186/s40359-017-0183-2. 